\title{
Purificación parcial de péptidos del veneno de escorpión Hadruroides charcasus (Karsch, 1879) con actividad antimicrobiana
}

\author{
Partial purification of peptides from the scorpion venom Hadruroides charcasus (Karsch, \\ 1879) with antimicrobial activity
}

Orlando Pérez-Delgado ${ }^{1}$, Clara Andrea Rincon-Cortés ${ }^{2}$, Nohora Angélica Vega-Castro ${ }^{3}$, Edgar Antonio Reyes-Montaño ${ }^{4}$, Marcela Gómez-Garzón ${ }^{5}$

DOI. 10.21931/RB/2021.06.03.6

Resumen: Los venenos de muchas especies de escorpiones son fuentes ricas en componentes biológicamente activos, como los péptidos antimicrobianos, biomoléculas que aún no han sido estudiados del veneno de Hadruroides charcasus. El objetivo de este artículo es evaluar la actividad antimicrobiana de los péptidos parcialmente purificados del veneno del escorpión Hadruroides. asus. A partir de 15,46 mg de proteína total del veneno del escorpión H. charcasus se purificaron parcialmente sus péptidos por medio de cromatografía de filtración en gel empleando sephadex G-75, consecutivo a una cromatografía de intercambio iónico en CM-Sephadex C-25. El peso molecular estimado de los péptidos se determinó mediante electroforesis PAGE-SDS-Tris-Tricina al 15\% y la evaluación de la actividad antibacteriana y antifúngica se empleó el método de microdilución y Kirby-Bauer con cepas de Escherichia coli ATCC 25922, Staphylococcus aureus ATCC 29213, Pseudomonas aeruginosa ATCC 27853 y Candida albicans ATCC 10231. En la cromatografía de filtración en gel se obtuvieron 5 fracciones, de lo cual, la fracción IV presentó una concentración mínima inhibitoria de 3,6 mg/mL en S. aureus ATCC 29213 y en C. albicans ATCC 10231. De la cromatografía de intercambio se obtuvieron 7 fracciones, destacando la fracción OPDIV-5 con péptidos de 4 kDa; 5 kDa; 5,5 kDa y 6,4 kDa que presentó actividad antimicrobiana frente S. aureus ATCC 29213, E. coli ATCC 25922, P. aeruginosa ATCC 27853, C. albicans ATCC 10231. El veneno del escorpión H. charcasus presenta péptidos de naturaleza catiónica con actividad antibacteriana y antifúngica, según su actividad en las cepas evaluadas.

Palabras clave: Antibacteriano, antifúngico, cromatografía de filtración, cromatografía de intercambio iónico, electroforesis.

\begin{abstract}
Venoms of many scorpion species are rich sources of biologically active components, such as antimicrobial peptides, biomolecules that have not yet been studied from Hadruroides charcasus venom. The aim of this article is to evaluate the antimicrobial activity of partially purified peptides from the venom of the scorpion Hadruroides charcasus. asus. From $15.46 \mathrm{mg}$ of total protein from H. charcasus scorpion venom, its peptides were partially purified by gel filtration chromatography using sephadex G-75, followed by ion exchange chromatography on CM-Sephadex C-25. The estimated molecular weight of the peptides was determined by $15 \%$ PAGE-SDSTris-Tricine electrophoresis and the evaluation of antibacterial and antifungal activity used the microdilution and Kirby-Bauer method with strains of Escherichia coli ATCC 25922, Staphylococcus aureus ATCC 29213, Pseudomonas aeruginosa ATCC 27853 and Candida albicans ATCC 10231. In gel filtration chromatography, 5 fractions were obtained, of which fraction IV showed a minimum inhibitory concentration of $3.6 \mathrm{mg} / \mathrm{mL}$ in S. aureus ATCC 29213 and C. albicans ATCC 10231. From the exchange chromatography, 7 fractions were obtained, highlighting the OPDIV-5 fraction with peptides of $4 \mathrm{kDa} 5 \mathrm{kDa} ; 5.5 \mathrm{kDa}$ and $6.4 \mathrm{kDa}$ that presented antimicrobial activity against S. aureus ATCC 29213, E. coli ATCC 25922, P. aeruginosa ATCC 27853, C. albicans ATCC 10231. H. charcasus scorpion venom presents peptides of cationic nat
\end{abstract}

Key words: Antibacterial, antifungal, filtration chromatography, ion exchange chromatography, electrophoresis.

\section{Introducción}

La resistencia a los antimicrobianos es un problema de salud mundial tanto en medicina humana como veterinaria donde los agentes antimicrobianos han sido ampliamente utilizados para tratar enfermedades bacterianas, por ejemplo, Escherichia coli es un microorganismo comensal común en personas y animales con ciertas cepas que son patógenas y se consideran excelentes indicadores de resistencia a los antimicrobianos $^{1}$

También se conoce que Pseudomonas aeruginosa es una bacteria metabólicamente versátil que puede causar una amplia gama de infecciones oportunistas graves y a menudo muestran resistencia adaptativa, debido al estado de crecimiento de la bacteria en el paciente, incluida la capacidad del microorganismo para crecer como biopelícula y además que conducen a la mortalidad ${ }^{2}$. Otro de los ejemplos son reportes de Staphylococcus aureus, que está relacionada con su capacidad para adquirir y diseminar determinantes resistentes a los antimicrobianos en la naturaleza. En un estudio donde fueron positivos para S. aureus por PCR, más del 50\% mostraron resistencia fenotípica a la meticilina, como también resistencia registrada a ampicilina y penicilina en un 96,7\%, rifampicina y clindamicina $80 \%$, oxacilina $73,3 \%$ y eritromicina $70 \%$. También S. aureus reveló susceptibilidad variable a imipenem $96,7 \%$, levofloxacina $86,7 \%$, cloranfenicol $83,3 \%$, cefoxitina $76,7 \%$, ciprofloxacina $66,7 \%$, gentamicina $63,3 \%$, tetraciclina y sulfametoxazol-trimetoprima $56,7 \%$ y vancomicina con doxiciclina en

\footnotetext{
¿Universidad Señor de Sipán

${ }^{2}$ Universidad de Ciencias Aplicadas y Ambientales.

${ }^{3}$ Universidad Nacional de Colombia.

${ }^{4}$ Fundación Universitaria de Ciencias de la Salud.

Corresponding author: operezd@gmail.com
} 
un $50 \%{ }^{3}$. Por otro lado, pero casos relacionados a las bacterias, son las infecciones fúngicas, las cuales han aumentado significativamente, lo que contribuye a la morbilidad y la mortalidad, como es el caso de Candida albicans caracterizado como un patógeno oportunista, a pesar de las terapias antifúngicas, ha llegado a ser letal por el aumento de la resistencia antimicrobiana, con una resistencia significativa frente a fluconazol e itraconazol a diferencia de otros antifúngicos que se reporta sensibilidad tales como para ketoconazol y anfotericina $B^{4}$

Razones en las que actualmente se ha identificado un mecanismo intrínseco y la adquisición de resistencia antimicrobiana en muchas cepas bacterianas que son de gran importancia clínica por tal motivo ha puesto en grave peligro el uso de antibióticos y también ha provocado la propagación de microbios que son resistentes a los medicamentos efectivos de primera elección ${ }^{5}$, Tal es así que la bacterias tanto gram positivas como gram negativas han logrado esta resistencia gracias a elementos genéticos móviles capaces de insertarse y transferirse de bacteria a bacteria ${ }^{6}$. Casos que han derivado el desarrollo de varios estudios experimentales, donde se ha logrado determinar que principios bioactivos tales como péptidos presentes en los venenos de escorpión logran desarrollar actividad antimicrobiana in vitro frente a diferentes microorganismos de importancia en el área de salud. Identificando actividades, independiente tanto de la especie de escorpión como del microorganismo evaluado. Uno de los reportes, es a partir del veneno del escorpión Tityus discrepans con una amplia actividad antibacteriana frente a bacterias Gram positivas y Gram negativas ${ }^{7}$, mientras que los venenos de los escorpiones Urodacus yaschenkoi martensii y Buthus martensii y Androctonus amoeruxi son más efectivos con bacterias Gram positivas, entre ellas $S$. aureus meticilino resistente (MRSA), siendo una cepa multidrogorresistente ${ }^{10}$. Y el veneno de Vaejovis punctatus con actividad antifúngica ${ }^{11}$. Características tomadas en cuenta para determinar si el veneno de $H$. charcasus posee efecto antimicrobiano sobre microorganismos de interés clínico, se planteó en el presente estudio cuyo objetivo es, evaluar la actividad antimicrobiana de péptidos aislados del veneno del escorpión H. charcasus frente E. coli ATCC 25922, S. aureus ATCC 29213, P. aeruginosa ATCC 27853 y C. albicans ATCC 10231.

\section{Materiales y métodos}

\section{Escorpiones y veneno}

Los especímenes de $\mathrm{H}$. charcasus fueron recolectados en el distrito de Chongoyape, ubicado en la latitud 6우 37' 40.0" y longitud 792 25' 21.4", Chiclayo (Perú), la identificación de la especie por el Dr. José Ochoa Cámara, de la Universidad Nacional de San Antonio Abad del Cusco el veneno se obtuvo por estimulación eléctrica ${ }^{12}$, luego se recolectó en tubos eppendorf, se liofilizó y se conservó a $-20{ }^{\circ} \mathrm{C}$ hasta su uso, de 60 escorpiones se obtuvo 1,2 $\mathrm{ml}$ de veneno soluble.

\section{Purificación de péptidos del veneno de $\boldsymbol{H}$. charcasus}

Para la cromatografía de filtración en gel, se aplicaron 15,46 mg de proteína del veneno a una columna de Sephadex G-75 $(1,5 \mathrm{~cm} \times 50 \mathrm{~cm})$ en presencia de buffer acetato de amonio al $20 \mathrm{mmol} / \mathrm{l}, \mathrm{pH}$ 4,7, a una velocidad de flujo de 500 $\mu \mathrm{l} / 4 \mathrm{~min}^{13}$, las fracciones con actividad antimicrobiana, luego se separaron por cromatografía de intercambio iónico, en una columna de carboximetilcelulosa (CM-Sephadex C-25; $2 \mathrm{~cm} \times 9$ $\mathrm{cm})$, equilibrada y desarrollada en presencia de buffer acetato de amonio, $\mathrm{pH} 4,7$; con un gradiente continuo de $0 \mathrm{~mol} / \mathrm{l}$ a 0,5 $\mathrm{mol} / \mathrm{ll}^{14}$

\section{Composición del veneno y sus fracciones mediante método de electroforesis PAGE-SDS-Tricina}

En condiciones desnaturalizantes fue realizada la electroforesis SDS-PAGE basados en el método de Schägger y Von Jagow $^{15}$, en una corrida a corriente constante y en gel de poliacrilamida al 15,0\%. Para el buffer de corrida se empleó solución de tris-tricina-dodecil sulfato de sodio y como estándar de proteínas de rango bajo pre-teñidas (2 a 40 kDa) Thermo Scientific ${ }^{\top M}$.

Los geles obtenidos, se revelaron por el método de tinción con plata ${ }^{16}$. Finalmente, se registraron digitalmente los geles en el equipo ChemiDocTM XRS, Bio-Rad.

\section{Actividad antimicrobiana del veneno $\mathrm{H}$. charcasus}

En esta parte de la metodología, se empleó el método de microdilución ${ }^{17,18}$ con la fracción IV y V de la cromatografía de filtración, se realizaron diluciones dobles seriadas, con un total de siete concentraciones, para la fracción IV de 3,6 mg/ml hasta $0,056 \mathrm{mg} / \mathrm{mL}$ y para la fracción $V$ de $6,3 \mathrm{mg} / \mathrm{mL}$ hasta $0,098 \mathrm{mg} / \mathrm{ml}$.

Para la preparación del inóculo, se emplearon cepas de $E$. coli ATCC 25922, S. aureus ATCC 29213, P. aeruginosa ATCC 27853 y C. albicans ATCC 10231, se realizaron ensayos por triplicado sembrando $50 \mu \mathrm{L}$ del inóculo conteniendo $5 \times 10^{4}$ UFC/ $\mathrm{ml}$ en cada pocillo, luego se repartieron $50 \mu \mathrm{l}$ de cada dilución, después se incubaron a $37 \stackrel{\circ}{ } \mathrm{C}$ durante 24 horas. Los controles positivos fueron caldo más inóculo, y los controles negativos solo caldo. El crecimiento del control positivo se determinó por un botón de crecimiento de $\geq 2 \mathrm{~mm}$ o una turbidez definida. Se empleó el método Kirby-Bauer ${ }^{19}$, para la sensibilidad antimicrobiana de las fracciones de la cromatografía de intercambio iónico, en las placas de agar Mueller-Hinton se sembraron con un inóculo de 1 a $2 \times 10^{8} \mathrm{UFC} / \mathrm{ml}$.

El análisis de los datos se realizó usando el complemento MegaStat para Excel, con análisis varianza para determinar si los componentes proteicos purificados del veneno y las concentraciones empleadas si influyeron en el crecimiento microbiano.

\section{Resultados}

A partir de 15,46 mg de proteínas total del veneno de $H$. charcasus, se analizaron por el método de cromatografía de filtración en gel Sephadex G-75, obteniendo cinco fracciones bien definidas de la cromatografía de filtración Sephadex G-75 (Figura 1), en el análisis del gel de electroforesis de Poliacrilamida (PAGE-SDS-Tricina), presentó un número variable bandas proteicas para cada uno de las fracciones purificadas, asimismo reveló para la fracción IV la presencia de siete bandas con rango de pesos moleculares de 9,8 kDa, 12 kDa, 15,1 kDa, 18,3 kDa, 24,3kDa, 30,2 kDa y 37,5kDa y para la fracción V se obtuvieron 6 bandas con rango de peso entre 5,1 kDa, 5,7 kDa, 7,9 kDa, 8,5 kDa, 10,7 kDa y 12,2 kDa respectivamente (Figura 2).

En la evaluación de la actividad antimicrobiana se muestra en la fracción IV presentó una concentración mínima inhibitoria (CMI) de 3,6 mg/mL para las cepas de S. aureus ATCC 29213 y C. albicans ATCC 10231 y para las cepas de E. coli ATCC 25922 y P. aeruginosa ATCC 27853 no presentó inhibición total de 


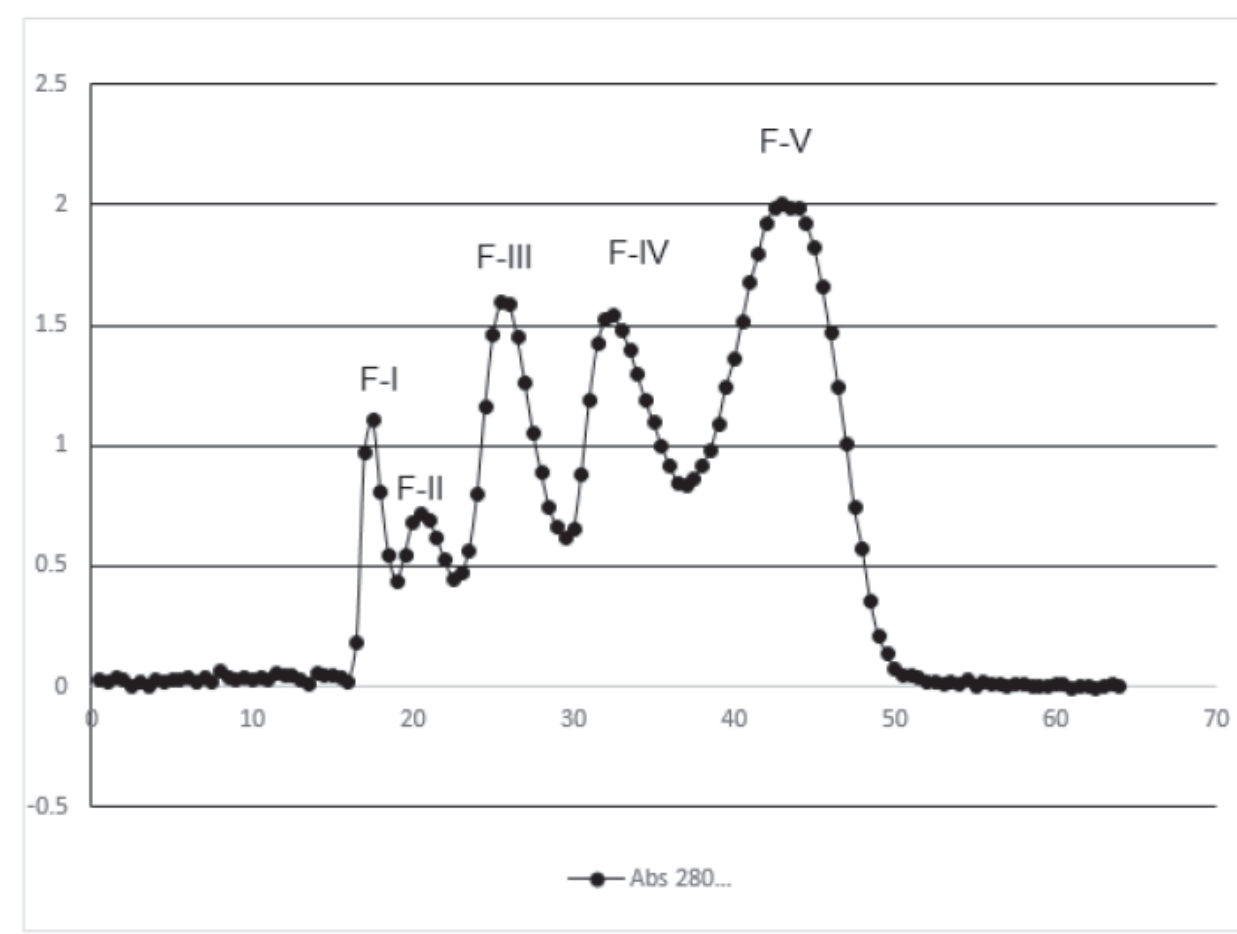

Figure 1. Purificación de las proteínas del veneno de H. charcasus por cromatografía de filtración en columna Sephadex G-75 con las fracciones (F-I, F-II, F-III, F-IV y F-V).

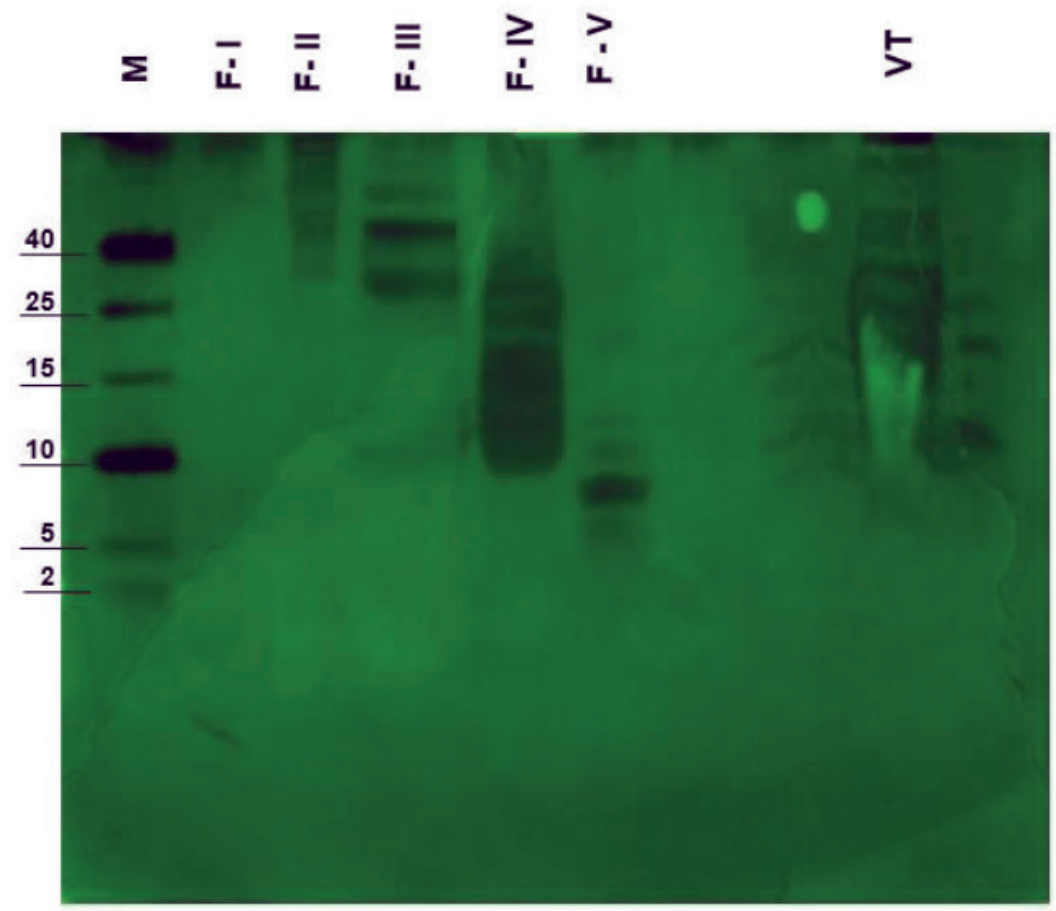

Figura 2. Electroforesis en gel de poliacrilamida con SDS - Tricina (PAGE-SDS-Tricina) de las proteínas purificadas, en el carril M marcador de peso molecular $(2-40 \mathrm{KDa})$ (marcador de peso molecular) y fracciones de proteínas (FI - FV) y veneno total (VT).

crecimiento a las concentraciones empleadas respectivamente (Figura 3), para la fracción $\vee$ no presentó inhibición total de crecimiento microbiano (Figura 4).

En la figura 5 se muestra el análisis de la separación de los péptidos por el método de cromatografía de intercambio iónico Sephadex C-25, se recuperaron al menos siete fracciones (OPDIV-1 a OPDIV-7), de los cuales al evaluar la actividad antimicrobiana mediante el método Kirby-Bauer, las cepas de E. coli ATCC 25922, S. aureus ATCC 29213, C. albicans ATCC 10231 y $P$. aeruginosa ATCC 27853 presentaron sensibilidad frente a la fracción OPDIV-5 a concentración de $1 \mu \mathrm{g} / \mathrm{mL}$ (tabla 1) y en el análisis de PAGE-SDS-Tricina, la fracción OPDIV-5 presentó cuatro bandas de pesos moleculares de 7,5 kDa, 6,4 kDa, 5,5 kDa y $4 \mathrm{kDa}$ (Figura 6).

\section{Discusión}

Actualmente los escorpiones son bien conocidos por sus picaduras peligrosas y sus venenos se han aplicado en la medicina tradicional, principalmente en Asia y África y se ha convertido en una valiosa fuente de moléculas biológicamente activas desde nuevos antibióticos hasta posibles terapias contra el cáncer ${ }^{20}$, además de acuerdo a los reportes publicados el veneno de escorpión posee una fuente abundante péptidos antimicrobianos nuevos y potentes tanto como naturales o modificados químicamente con la finalidad ampliar su capacidad antibacteriana, antifúngica, antiparasitaria ${ }^{21,22}$. Los péptidos antimicrobianos son uno de los componentes del sistema inmune innato en una amplia gama de organismos eucariotas como hu- 


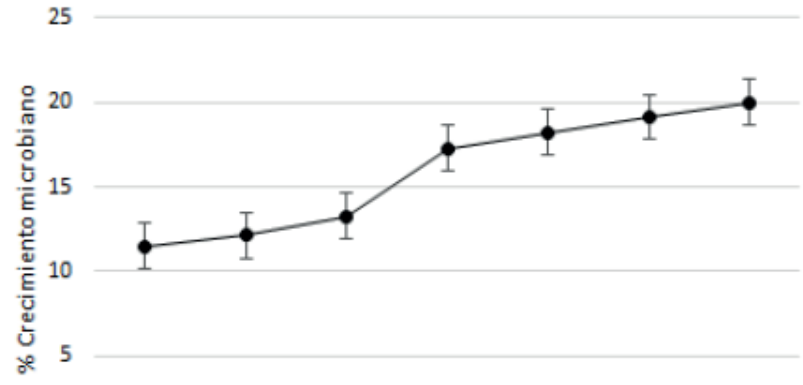

0

$[3.6] \quad[1.8] \quad[0.9] \quad[0.45] \quad[0.225] \quad[0.1125] \quad[0.056]$

Concentraciones $\{\mathrm{mg} / \mathrm{mL}\}$

$\longrightarrow$ Escherichiacoli ATCC 25922

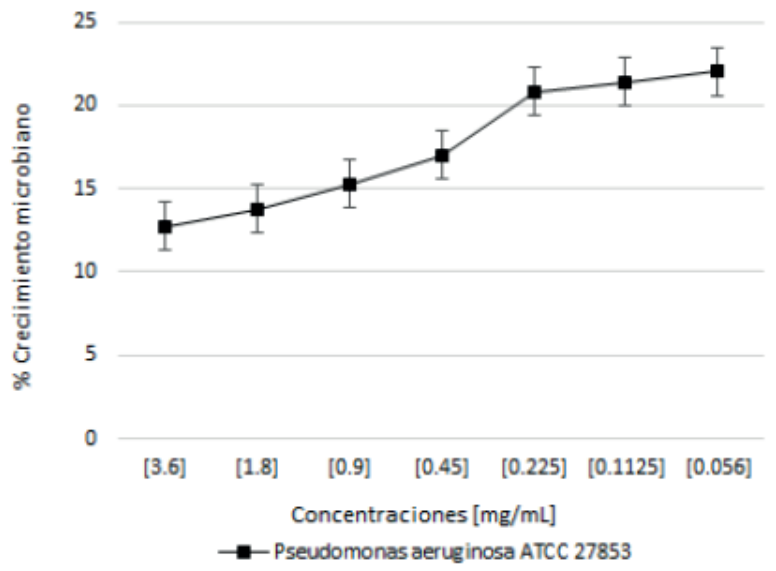

Figura 3. Actividad antimicrobiana de la fracción IV frente Escherichia coli ATCC 25922, Staphylococcus aureus ATCC 29213, Pseudomonas aeruginosa ATCC 27853 y Candida albicans ATCC 10231.

manos, plantas e insectos y que juegan un papel vital en los primeros mecanismos de defensa inmune contra los patógenos ${ }^{23}$.

En el presente estudio se logró determinar la presencia péptidos antimicrobianos del veneno de $H$. charcasus esto es algo propio del veneno de los escorpiones tal como se evidencia de otras especies de escorpiones que demostraron e inclusive aislar péptidos con potencial acción antimicrobiana como las pantininas de Pandinus imperator ${ }^{24}$, los péptidos TsAP-1 y TsAP-2 de Tityus serrulatus ${ }^{25}$ péptidos del veneno de Scorpio maurus palmatus ${ }^{26}$ y los péptidos HsAp del escorpión Heterometrus spinifer ${ }^{27}$

Además, en el presente trabajo mediante el empleo de dos métodos cromatográficos, uno de filtración en gel y otro de intercambio iónico permitió separar las proteínas en fracciones. Cuando el veneno crudo se separó por columna Sephadex G-75, se obtuvieron 5 fracciones, siendo evidente la fracción IV con actividad antibacteriana frente a $S$. aureus y antifúngica frente a $C$. albicans. Esta fracción relacionada con su actividad antimicrobiana fue purificada y analizada en CM Sephadex C-25, obteniendo 7 fracciones, de los cuales la quinta fracción denominada OPDIV-5 contiendo péptidos con pesos moleculares de $7,5 \mathrm{kDa}, 6,4 \mathrm{kDa}, 5,5 \mathrm{kDa}$ y $4 \mathrm{kDa}$ mediante el método Kirby-Bauer se encontró actividad frente E. coli, S. aureus, P. aeruginosa y C. albicans.

Esto es característico de los péptidos antimicrobianos que por lo general presentan peso molecular por debajo de $8,5 \mathrm{kDa}$ como es el caso de las familias de las opiscorpinas del escorpión Opistophthalmus carinatus ${ }^{28}$, vejonina de Vaejovis mexi-

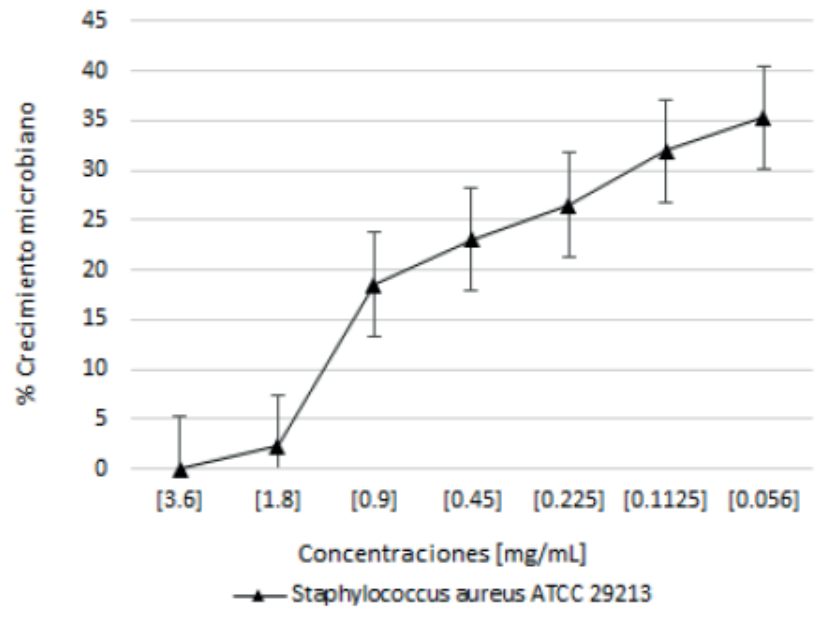

10

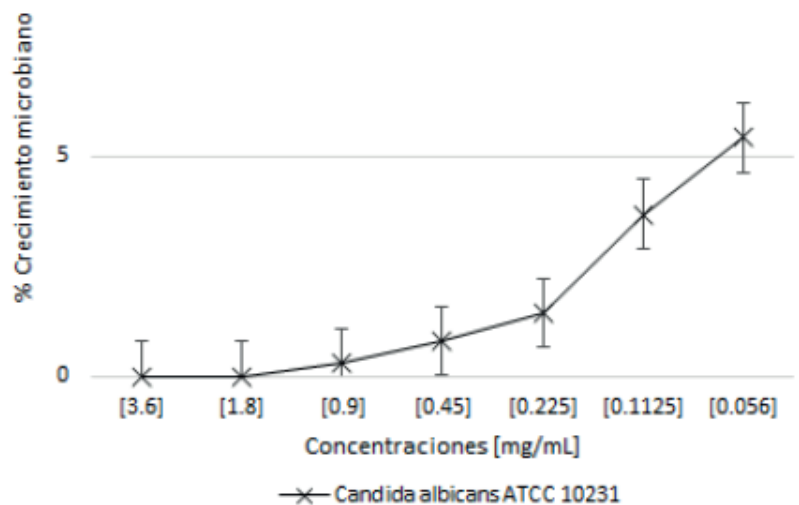

canus $^{29}$ Heterinas de Heterometrus spinifer ${ }^{30}$, demostrando su amplia acción antibacteriana y antifúngica.

La purificación parcial de la fracción OPDIV-5 conformado por cuatro péptidos con carga positiva que al interactuar con las cargas negativas de la CM Sephadex C-25, solo se pudo eluir aumentando la concentración del acetato de amonio, además, presentó actividad antibacteriana como actividad antifúngica. esto se debió al empleó mayor concentración del buffer acetato de amonio con la finalidad de recolectar péptidos de naturaleza catiónica y con mayor carga.

Los péptidos antimicrobianos del veneno de escorpión son péptidos anfipáticos cargados positivamente, se manifiesta en los péptidos meuVAP-6, meuAP-18-1 y meuPep34 de Mesobuthus eupeus ${ }^{31}$, inclusive en los péptidos VpAmp de Vaejovis punctatus, por presentar acción contra bacterias gram positivas y contra $C$. albicans y frente a bacterias gram negativas ${ }^{32}$, similar fue para los péptidos UyCT de Urodacus yaschenkoi por tener predilección por bacterias gram positivas ${ }^{33}$, péptido antibacteriano de Centruroides margaritatus ${ }^{34}$.

\section{Conclusiones}

El veneno de $H$. charcasus, posee péptidos de naturaleza catiónica, con capacidad antimicrobiana frente a microorganismos patógenos de importancia clínica. 

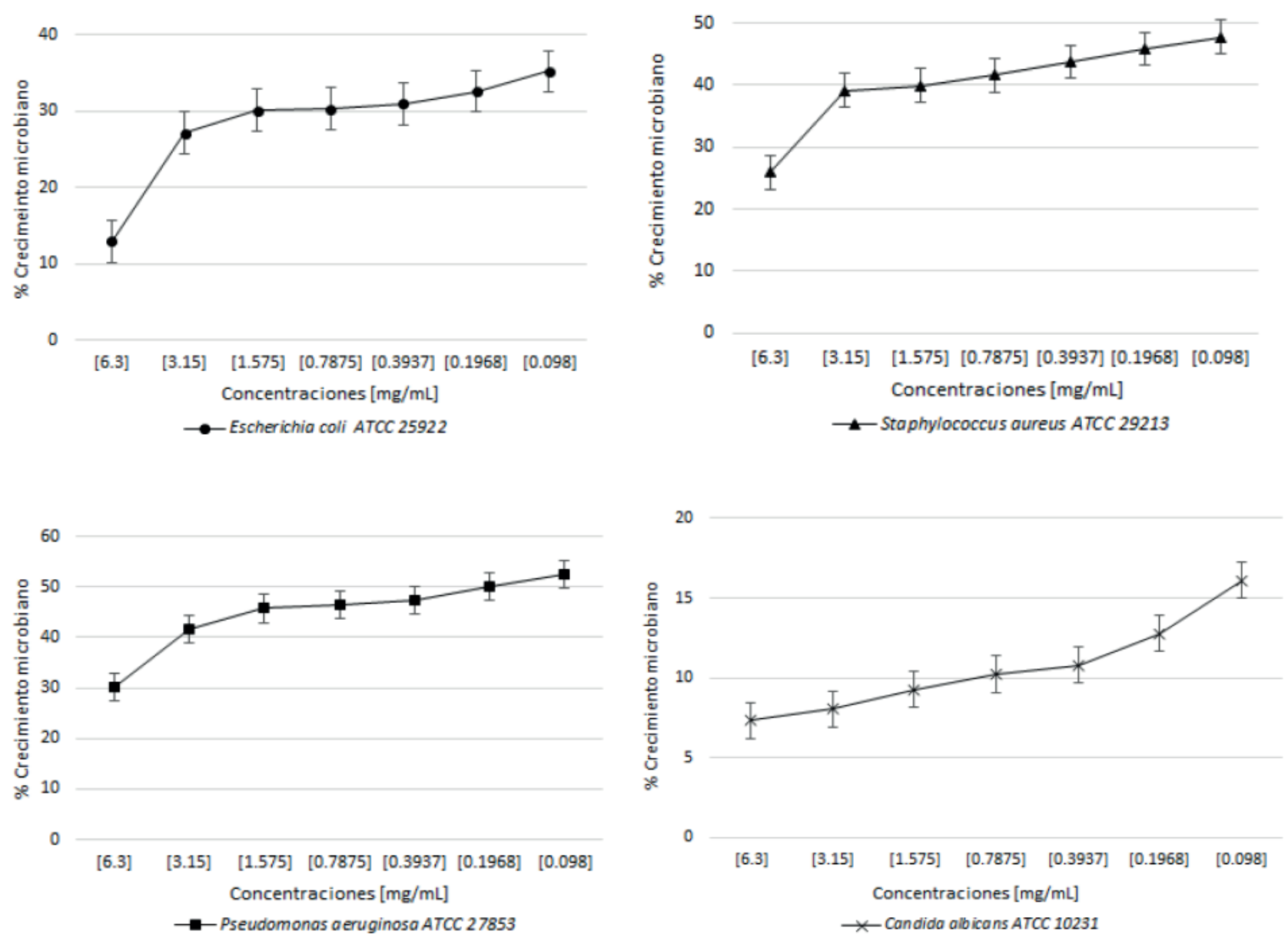

Figura 4. Actividad antimicrobiana de la fracción V frente a Escherichia coli ATCC 25922, Staphylococcus aureus ATCC 29213, Pseudomonas aeruginosa ATCC 27853 y Candida albicans ATCC 10231.

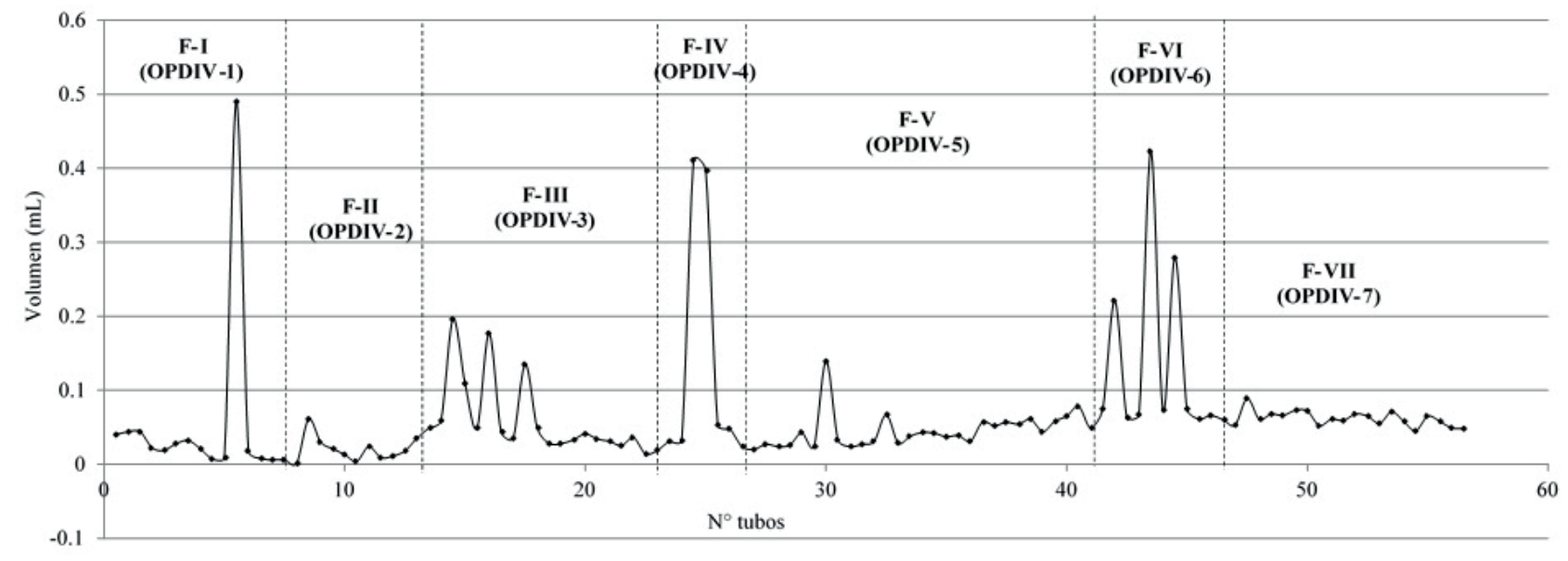

Figura 5. Purificación de la fracción IV (Sephadex G-75) con acción antimicrobiana, en cromatografía de intercambio iónico Carboximetilcelulosa Sephadex C-25.

\section{Agradecimientos}

Agradecimiento al Grupo de Investigación en Proteínas de la Universidad Nacional de Colombia sede en Bogotá, por la oportunidad para el desarrollo del trabajo de investigación.

\section{Conflicto de interés}

Los autores declaramos no presentar conflictos de interés potenciales con respecto a la investigación, autoría y/o publicación de este artículo. 


\begin{tabular}{|l|c|c|c|c|}
\hline Fracciones/ & $\begin{array}{c}\text { E. coli ATCC } \\
25922\end{array}$ & $\begin{array}{c}\text { S. aureus } \\
\text { ATCC 29213 }\end{array}$ & $\begin{array}{c}\text { P. aeruginosa } \\
\text { ATCC 27853 }\end{array}$ & $\begin{array}{c}\text { C. albicans } \\
\text { ATCC } 10231\end{array}$ \\
\hline concentraciones & - & - & - & - \\
\hline OPDIV-1 $(4,96 \mathrm{ug} / \mathrm{mL})$ & - & - & + & - \\
\hline OPDIV -2 $(14,5 \mathrm{ug} / \mathrm{mL})$ & - & - & - & - \\
\hline OPDIV -3 $(3,42 \mathrm{ug} / \mathrm{mL})$ & - & - & - & + \\
\hline OPDIV -4 $(3,02 \mathrm{ug} / \mathrm{mL})$ & + & + & + & + \\
\hline OPDIV -5 $(1 \mathrm{ug} / \mathrm{mL})$ & - & - & - & + \\
\hline OPDIV -6 $(7,72 \mathrm{ug} / \mathrm{mL})$ & + & - & - & + \\
\hline OPDIV -7 $(2,24 \mathrm{ug} / \mathrm{mL})$ & \multicolumn{2}{|c|}{ Con actividad +; Sin actividad - } & \\
\hline
\end{tabular}

Tabla 1. Sensibilidad antimicrobiana de las fracciones de la cromatografía de intercambio iónico (OPDIV-1 a OPDIV-7) frente a Escherichia coli ATCC 25922, Staphylococcus aureus ATCC 29213, Pseudomonas aeruginosa ATCC 27853 y Candida albicans ATCC 10231.

Figura 6. Electroforesis en gel de poliacrilamida con SDS - Tricina (PAGE-SDS-Tricina) de las proteínas purificadas de la fracción IV de la Cromatografía de intercambio iónico Sephadex C-25, en el carril M marcador de peso molecular (2 - 40 kDa) y fracción OPDIV-5

\section{Referencias bibliográficas}

1. Yassin AK, Gong J, Kelly P, Lu G, Guardabassi L, Wei L, et al. Antimicrobial resistance in clinical Escherichia coli isolates from poultry and livestock, China. PLoS ONE. 2017; 12(9): e0185326. DOI: http://dx.doi.org/10.1371/journal.pone.0185326

2. Gellatly SL, Hancock REW. Pseudomonas aeruginosa: new insights into pathogenesis and host defenses. Path and Dis. 2013; 67: 159 - 173. DOI: http://dx.doi.org/10.1111/2049-632X.12033

3. Akanbi OE, Njom HA, Fri J, Otigbu AC, Clarke AM. Antimicrobial Susceptibility of Staphylococcus aureus Isolated from Recreational Waters and Beach Sand in Eastern Cape Province of South Africa. Int. J. Environ. Res. Public Health. 2017; 14: 1001. DOl: http://dx.doi.org/10.3390/ijerph14091001

4. Zaidi KU, Mani A, Parmar R, Thawani V. Antifungal Susceptibility Pattern of Candida albicans in Human Infections. Op. Biol. Scie. J. 2018; 4: 1 - 6. DOl: http://dx.doi. org/10.2174/2352633501804010001

5. Rayamajhi N, Bin Cha S, Yoo HS. Antibiotics Resistances: Past, Present and Future. J. Biomed Res. 2010; 11(2): 65 - 80. Disponible en: http://www.jbr.or.kr/journal/article.php?code=5527

6. Partridge SR, Kwong SM, Firth N, Jensen SO. Mobile genetic elements associated with antimicrobial resistance. Clin Microbiol Rev 2018; 31: e00088-17. DOl: https://doi.org/10.1128/ CMR.00088-17

7. Díaz P, D'Suze G, Salazar V, Sevcik C, Shannon JD, Sherman NE, Fox JW. Antibacterial activity of six novel peptides from Tityus discrepans scorpion venom. A fluorescent probe study of microbial membrane $\mathrm{Na}$ + permeability changes. Toxicon. 2009; 54: 802 - 817. DOI: http://dx.doi.org/10.1016/j.toxicon.2009.06.014

8. Luna-Ramírez K, Quintero-Hernández V, Vargas-Jaimes L, Batista CVF, Winkel, KD, Possani LD. Characterization of the venom from the Australian scorpion Urodacus yaschenkoi: Molecular mass analysis of components, cDNA sequences and peptides with antimicrobial activity. Toxicon. 2013; 63: 44 - 54. DOI: http:// dx.doi.org/10.1016/j.toxicon.2012.11.017

9. de la Salud R, Ross M, Luque LE. Synthesis of analogs of peptides from Buthus martensii scorpion venom with potential antibiotic activity. Peptides. 2015; 68: 228 - 232. DOl: http://dx.doi. org/10.1016/j.peptides.2014.10.008

10. Almaaytah A, Farajallah A, Abualhaijaa A, Al-Balas Q. A3, a Scorpion Venom Derived Peptide Analogue with Potent Antimicrobial and Potential Antibiofilm Activity against Clinical Isolates of Multi-Drug Resistant Gram Positive Bacteria. Molecules. 2018; 23: 1603. DOI: http://dx.doi.org/10.3390/molecules23071603

11. Ramírez S, Jiménez JM, Rivas B, Corzo G, Possani LD, Becerril B, Ortiz E. Peptides from the scorpion Vaejovis punctatus with broad antimicrobial activity. Peptides. 2015; 73: 51-59. DOl: http://dx. doi.org/10.1016/j.peptides.2015.08.014 
12. Pérez-Delgado O, Espinoza-Vergara M, Castro-Vega N, Reyes-Montaño E. Evaluación preliminar de actividad antibacteriana in vitro del veneno de escorpión Hadruroides charcasus (Karsch, 1879) contra Pseudomonas aeruginosa y Staphylococcus aureus. Rev C Méd HNAAA, 2019; 12(1): 6 - 12. DOl: https:// doi.org/10.35434/rcmhnaaa.2019.121.477

13. Possani LD, Dent MAR, Martin BM, Maelicke A, Svendsen I. The amino terminal sequence of several toxins from the venom of the Mexican scorpion Centruroides noxius Hoffmann. Carlsberg Res. Commun. 1981; 46: 207-214. DOl: https://doi.org/10.1007/ BF02906498

14. Ramirez-Dominguez ME, Olamendi-Portugal T, Garcia U, Garcia C, Arechiga H, Possani LD. Cnl1, the first example of a scorpion toxin that is a true blocker of $\mathrm{Na}(+)$ currents in crayfish neurons. The Journal of experimental biology. 2002; 205(Pt 6):869-76. Disponible en: https://jeb.biologists.org/content/205/6/869

15. Schägger, H.; von Jagow, G. 1987. Tricine-sodium dodecyl sulfate polyacrylamide gel electrophoresis for the separation of proteins in the range from 1-100 kDalton. Anal. Biochem. 166: 368 - 379. DOl: https://doi.org/10.1016/0003-2697(87)90587-2

16. Shevchenko A, Wilm M, Vorm O, Mann M. Mass Spectrometric Sequencing of Proteins from Silver-Stained Polyacrylamide Gels Anal. Chem. 1996; 68: 850 -858. DOl: https://doi.org/10.1021/ ac950914h

17. National Committee for Clinical Laboratory Standards. Methods for dilution antimicrobial susceptibility tests for bacteria that grow aerobically. Document M07-A10. 2015. NCCLS, Wayne, PA. Recuperado a partir de: https://clsi.org/

18. Clinical and Laboratory Standars Institute. Reference Method for Broth Dilution Antifungal Susceptibility Testing Yeasts. Aprproved Estándar - tercera edición 2008 (28)14: 1 - 25. M27-A3. Recuperado a partir de: https://clsi.org/

19. Clinical and Laboratory Standars Institute. Performance Standards for Antimicrobial Disk Susceptibility Tests Document M02-A13. 2018. NCCLS, Wayne, PA. Recuperado a partir de: https://clsi.org/

20.Ortiz E, Gurrola GB, Ferroni E, Possani LD. Scorpion venom components as potential candidates for drug Development Toxicon. 2015; 93: 125e135. DOI: http://dx.doi.org/10.1016/j.toxicon.2014.11.233

21. Luna-Ramirez K, Tonk M, Rahnamaeian M, Vilcinskas A. Bioactivity of Natural and Engineered Antimicrobial Peptides from Venom of the Scorpions Urodacus yaschenkoi and U. manicatus. Toxins. 2017; 9 (22): 1 -12. DOl: http://dx.doi.org/10.3390/toxins9010022

22. Borges A, Delgado O, Silva S, Bravo J, Velasco E, Rojas L, De Sousa L. Aislamiento y caracterización de un péptido del veneno de Tityus gonzalespongai (scorpiones, buthidae) con actividad sobre promastigotes de Leishmania (Leishmania) mexicana. Saber. 2013; 25 (4): 399 - 413. Disponible en: http://ve.scielo.org/scielo. php?script=sci_arttext\&pid=S1315-01622013000400008\&L$\mathrm{ng}=\mathrm{es}$

23. arazi S. Scorpion venom as antimicrobial peptides (AMPs): A review article. Inter. arab. J. anti. age. 2015; 5(3): 1-5. Disponible en: https://imed.pub/ojs/index.php/IAJAA/article/view/1384/1134
24.Zeng CC, Zhou L, Shi W, Luo X, Zhang L, Nie Y, Wang J, Wu Sh, $\mathrm{Cao} \mathrm{B}, \mathrm{Cao} \mathrm{H}$. Three new antimicrobial peptides from the scorpion Pandinus imperator. Peptides. 2013; 45: 28-34. DOl: http://dx.doi. org/10.1016/j.peptides.2013.03.026

25. Guo X, Ma Ch, Du Q, Wei R, Wang L, Zhou M, Chen T, Shaw C. Two peptides, TsAP-1 and TsAP-2, from the venom of the Brazilian yellow scorpion, Tityus serrulatus: Evaluation of their antimicrobial and anticancer activities. Biochimie. 2013; 95: 1784 - 1794. DOI: http://dx.doi.org/10.1016/j.biochi.2013.06.003

26. Harrison PL, Abdel-Rahman MA, Strong PN, Tawfik MM, Miller K. Characterisation of three alpha-helical antimicrobial peptides from the venom of Scorpio maurus palmatus Toxicon. 2016;117: 30 - 6. DOI: http://dx.doi.org/10.1016/j.toxicon.2016.03.014

27. Nie Y, Chun X, Zeng XC, Yang Y, Luo F, Luo X, Wua S, Zhang L, Zhou J. A novel class of antimicrobial peptides from the scorpion Heterometrus spinifer. Peptides. 2012; 38: 389 - 394. DOI: http:// dx.doi.org/10.1016/j.peptides.2012.09.012

28. Zhu S, Tytgat J. The scorpine family of defensins: gene structure, alternative polyadenylation and fold recognition. Cell. Mol. Life Sci. 2004; 61: 1751e1763. DOl: https://doi.org/10.1007/s00018004-4149-1

29. Hernandez-Aponte CA, Silva-Sanchez J, Quintero-Hernandez V, Rodriguez-Romero A, Balderas C, Possani LD, Gurrola GB. Vejovine, a new antibiotic from the scorpion venom of Vaejovis mexicanus. Toxicon. 2011; 57: 84e92. DOI: https://doi.org/10.1016/j. toxicon. 2010.10 .008

30.Wu S, Nie Y, Zeng X-C, Cao H, Zhang L, Zhou L, Yang Y, Luo X, Liu $Y$. Genomic and functional characterization of three new venom peptides from the scorpion Heterometrus spinifer. Peptides. 2014; 53: 30-41. DOI: https://doi.org/10.1016/j.peptides.2013.12.012

31. Baradaran M. Jalali A, Naderi M, Galehdari H. A Novel Defensin-Like Peptide Associated with Two Other New Cationic Antimicrobial Peptides in Transcriptome of the Iranian Scorpion Venom. 2017; 21(3): 190 - 196. DOl: https://dx.doi.org/10.18869\%2Facadpub.ibj.21.3.190

32. Ramírez S, Jiménez JM, Rivas B, Corzo G, Possani LD, Becerril B, Ortiz E. Peptides from the scorpion Vaejovis punctatus with broad antimicrobial activity. Peptides. 2015; 73: 51-59. DOI: http://dx. doi.org/10.1016/j.peptides.2015.08.014

33. Luna K, Sani MA, Silva J, Jiménez JM, Reyna F, Winkel KD, Wright CE, Possani LD, Separovic F. Membrane interactions and biological activity of antimicrobial peptides from Australian scorpion. Bioch. et Bioph. Ac. 2014; 1838: 2140 - 2148. DOl: http://dx.doi. org/10.1016/j.bbamem.2013.10.022

34. Rivera C, Flores L, Pantigoso C, Escobar E. Aislamiento y caracterización de un péptido antibacteriano del veneno de Centruroides margaritatus. Rev. Per. Biol. 2010; 17(1): 129 -132. Disponible en: http://www.scielo.org.pe/pdf/rpb/v17n1/a16v17n1.pdf

Received: 10 marzo 2021

Accepted: 5 julio 2021 